\title{
Estrutura populacional de Drimys angustifolia Miers em um fragmento de Floresta Ombrófila Mista Alto-Montana, Urubici, SC
}

\author{
Population structure of Drimys angustifolia Miers in a fragment of Upper Montane Mixed \\ Ombrophilous Forest, Urubici, SC
}

\author{
Edilaine Duarte*, Ana Carolina da Silva, Pedro Higuchi, Jessica Thalheimer Aguiar, Monique Bohora \\ Schlickmann, Janaina Gabriela Larsen, Tarik Cuchi, Manoela Bez Vefago
}

Universidade do Estado de Santa Catarina, Lages, SC. Brasil. *Autor para correspondência: ediduarte10@gmail.com

\begin{abstract}
RESUMO
O presente estudo teve como objetivo caracterizar a estrutura populacional da espécie arbórea mais abundante em um fragmento de Floresta Ombrófila Mista Alto-Montana em Urubici, Santa Catarina, assim como determinar sua capacidade de regeneração natural na área desmatada adjacente ao fragmento florestal. Para a detecção da espécie mais abundante, foi realizado um levantamento florístico-estrutural no fragmento florestal, por meio da alocação de parcelas, onde todos os indivíduos com diâmetro a altura do peito $(\mathrm{DAP}) \geq 5 \mathrm{~cm}$ foram considerados adultos e inventariados. Para determinar a capacidade de regeneração natural da espécie selecionada na área desmatada adjacente, foram alocadas parcelas, onde os indivíduos considerados como regenerantes $(20 \mathrm{~cm}$ de altura até $5 \mathrm{~cm}$ de DAP) foram amostrados, nos anos de 2014 e 2016. Foi analisada a estrutura diamétrica e a distribuição espacial dos indivíduos adultos da espécie, e a estrutura hipsométrica, espacial e dinâmica dos indivíduos regenerantes. A espécie mais abundante no fragmento foi Drimys angustifolia Miers, que representou $26,4 \%$ dos indivíduos. A estrutura populacional dessa espécie obteve distribuição diamétrica tendendo à normalidade e distribuição espacial aleatória para os indivíduos adultos. O componente regenerante apresentou padrão de distribuição espacial agregado (2016) e distribuição hipsométrica normal somente em 2016. As taxas de dinâmica dos regenerantes indicaram aumento populacional. A elevada densidade desta espécie, associada às suas características estruturais, indicam boa adaptação no fragmento florestal e capacidade regenerativa condizente com o estágio sucessional da área em recuperação, com tendência ao aumento do número de indivíduos ao longo do tempo.
\end{abstract}

PALAVRAS-CHAVE: Drimys angustifolia Miers, estrutura populacional, regeneração natural.

\begin{abstract}
The present study had as objective to characterize the population structure of the most abundant arboreal species in a fragment of Upper Montane Mixed Ombrophilous Forest in Urubici, Santa Catarina, as well as determining the natural capacity of regeneration in the deforested area adjacent to the forest fragment. To detect the most abundant species, was realized a structural-floristic survey in the forest fragment, through the allocation of plots, in which all individuals with diameter at breast height (DBH) $\geq 5 \mathrm{~cm}$ were considered adults and inventoried. To determine the natural capacity of regeneration of the selected species in the deforested adjacent area, were allocated plots, where the individuals classified as regenerating $(20 \mathrm{~cm}$ of height to $5 \mathrm{~cm}$ of DAP) was sampled, in the years of 2014 and 2016. It was analyzed the diametric structure and the spatial distribution of the adults' individuals of the specie, and the hypsometric, spatial structures and dynamic of the regenerating individuals. Drimys angustifolia Miers was the most abundant species in the fragment, which represented $26.4 \%$ of the individuals. The population structure for this species obtained a diametric distribution tending to normality and random spatial distribution for the adults individuals. The regenerated component presented an aggregated spatial distribution pattern (2016) and normal hypsometrical distribution only in 2016. The dynamic rates of the reagents indicated a population increase. The great density for this species, associated with its structural characteristics, indicate a good adaption in this forest fragment and regenerative capacity consistent with the successional stage of the area under recovering, with a tendency to increase the number of individuals over time.
\end{abstract}

KEYWORDS: Drimys angustifolia Miers, population structure, natural regeneration. 


\section{INTRODUÇÃO}

Nas últimas décadas, a perda e a fragmentação de habitat têm contribuído para alterações na estrutura e na composição da biodiversidade dos biomas, podendo resultar na extinção de muitas espécies (MYERS 2000). No estado de Santa Catarina, 95\% dos remanescentes que constituem o bioma Mata Atlântica são formados por florestas secundárias (VIBRANS et al. 2011), compostas por mosaicos de vegetação em diferentes estágios de sucessão (SIMINSKI et al. 2013), sendo consequência de ações naturais ou antrópicas (SIMINSKI et al. 2004). Dentre as fitofisionomias do bioma que foram intensamente exploradas, destaca-se a Floresta Ombrófila Mista (FOM) (MARTINS-RAMOS et al. 2011, SILVA et al. 2012).

A FOM é constituída de quatro subformações, que se alteram ao longo de um gradiente altitudinal (IBGE 2012). Dentre estas, cita-se a FOM Alto-Montana, que ocorre em altitudes acima de $1.000 \mathrm{~m}$ no sul do Brasil (MARTINS-RAMOS et al. 2011, IBGE 2012). Essa formação apresenta baixa riqueza e endemismo (FALKENBERG 2003), devido a presença de fatores ambientais limitantes, como as baixas temperaturas, elevada umidade relativa do ar, presença frequente de neblina, menor incidência de radiação solar e a predominância de solos pouco profundos, ocasionando um ambiente restritivo para o estabelecimento de espécies (HIGUCHI et al. 2013).

Destaca-se que as formações alto-montanas apresentam importantes funções ecológicas, como, por exemplo, na proteção de encostas (BECKER et al. 2006), no estoque do carbono, no ciclo hidrológico (BOLSTAD \& VOSE 2005, MARTÍNEZ et al. 2009, SCHEER et al. 2011) e na conservação da biodiversidade, uma vez que abrigam elevado endemismo (BRUIJNZEEL 2000, FALKENBERG 2003). Devido às condições peculiares e restritivas destes ambientes, essa formação florestal pode apresentar elevado número de espécies endêmicas, com destaque para aquelas pertencentes às famílias Winteraceae, Elaeocarpaceae, Berberidaceae, Ericaceae e Cunoniaceae (KLEIN 1978).

Dessa forma, estudos sobre a estrutura diamétrica e hipsométrica de populações de espécies arbóreas desses ambientes são importantes para a compreensão do processo de estruturação e sucessão florestal, pois permitem inferências sobre a dinâmica florestal, assim como previsões futuras quanto ao desenvolvimento da comunidade vegetal (NASCIMENTO et al. 2001). Já a análise da distribuição espacial de espécies pode contribuir com o conhecimento da ocupação da espécie no espaço horizontal da floresta, indicando sua dispersão (DALE 2000). Assim, conhecer a estrutura populacional de espécies arbóreas é de grande importância na tomada de decisões e na adoção de técnicas para a conservação e o manejo (NASCIMENTO et al. 2001).

Considerando que informações ecológicas sobre a estrutura populacional e a capacidade regenerativa de espécies arbóreas são fundamentais para o manejo adequado de remanescentes florestais, visando uso sustentável e conservação, o presente estudo teve como objetivo caracterizar a estrutura populacional da espécie arbórea mais abundante em um fragmento de Floresta Ombrófila Mista AltoMontana no município de Urubici, Santa Catarina, assim como determinar sua capacidade de regeneração natural na área desmatada adjacente ao fragmento florestal.

\section{MATERIAL E MÉTODOS}

O estudo foi conduzido em um fragmento florestal localizado no Parque Nacional de São Joaquim em Urubici, SC, assim como em uma área adjacente a esse fragmento, desmatada no passado. Essas áreas, atualmente protegidas por terem sido incorporadas ao Parque, passaram por processos de corte seletivo e raso. O fragmento florestal se encontra, atualmente, em processo de sucessão avançada, enquanto que a área desmatada, que sofreu corte raso para a criação de gado, está em processo inicial de recuperação pós-distúrbio.

As áreas estão situadas a $1.628 \mathrm{~m}$ de altitude, nas coordenadas geográficas $28^{\circ} 05^{\prime} 41,5^{\prime \prime} \mathrm{S}$ e 49³0'14,71"W. De acordo com Köppen, o clima da região é do tipo Cfb, os solos são classificados como Neossolos Litólicos e Cambissolos, sendo pouco profundos, e o relevo varia de suave a fortemente ondulado. O fragmento é caracterizado como Floresta Ombrófila Mista Alto-Montana e, também, pode ser considerado como uma mata nebular, devido à presença frequente de neblina (FALKENBERG 2003, IBGE 2012). A área em estágio inicial de sucessão também era de floresta nebular e, há aproximadamente dez anos, se encontra em regeneração natural pós-desmatamento.

No fragmento florestal, foi realizado o levantamento florístico-estrutural do componente arbóreo adulto por meio da alocação de uma transecção de $20 \times 100 \mathrm{~m}$, subdividida em 20 parcelas de $10 \times 10 \mathrm{~m}$. Em cada parcela, foram identificados todos os indivíduos arbóreos com DAP (diâmetro a altura do peito, medido a $1,30 \mathrm{~m}$ do solo) $\geq 5 \mathrm{~cm}$. Foi quantificado o número de indivíduos de cada espécie e, para a espécie mais 
abundante, foi analisada a sua estrutura populacional. Para isso, foi analisada a estrutura diamétrica e a distribuição espacial. A estrutura diamétrica foi determinada por meio de histogramas de frequências, com base na distribuição dos indivíduos em classes de diâmetro $(n c)$, pelo método de Sturges: $n c=$ 1+3.33. $\log (n)$, em que $n=$ número de indivíduos. O intervalo de classes $(L)$ foi determinado por meio da razão entre a amplitude total e o número de classes: $L=R / n c$, em que: $R=$ amplitude (maior valor menos o menor valor da amostra). Para verificar a normalidade dos dados, aplicou-se o teste de Shapiro-Wilk. O padrão de distribuição espacial dos indivíduos da espécie mais abundante foi calculado por meio do Índice de Dispersão de Morisita (IM).

Para a área desmatada adjacente, foi estudado o componente regenerante nos anos de 2014 e 2016. Para isso, foi alocada uma transecção de $20 \times 100 \mathrm{~m}$ na área, subdividida em 20 parcelas permanentes de $10 \times 10 \mathrm{~m}$, sendo realizada a amostragem de todos os indivíduos com altura $\geq 20 \mathrm{~cm}$ e até $5 \mathrm{~cm}$ de DAP. Para o estudo do componente regenerante da espécie mais abundante do componente arbóreo adulto, no fragmento adjacente, foi mensurada a altura de todos os indivíduos amostrados da espécie, em ambos os anos de inventário.

Para a análise da dinâmica da população regenerante entre os anos de 2014 e 2016, nas mesmas parcelas foram contabilizados os indivíduos mortos, medidas a altura dos indivíduos sobreviventes e recrutados os indivíduos que atingiram o nível de inclusão de $20 \mathrm{~cm}$. Foram calculadas as taxas de recrutamento e mortalidade da espécie, por meio das equações descritas por SHEIL \& MAY (1996): $R=(1$ $(1-r / N t) 1 / t) \times 100$ e $M=(1-((N o-m) / N o) 1 / t) \times 100$, em que $M=$ taxa de mortalidade anual; $R=$ taxa de recrutamento anual; $N o=$ número inicial de árvores; $t=$ intervalo de tempo entre os inventários; $N t=$ número final de árvores depois de $t ; m=$ número de árvores mortas; $r=$ número de árvores recrutadas. Também foram analisadas as estruturas hipsométricas dos indivíduos para os anos de 2014 e 2016, utilizando-se a mesma fórmula da estrutura diamétrica dos indivíduos adultos citada acima.

A estrutura espacial dos regenerantes da espécie selecionada também foi obtida pelo Índice de Morisita, para o ano de 2016. Para a análise da estruturação espacial de segunda ordem dos indivíduos sobreviventes em 2016, foi utilizada a função $L$ univariada, com correção isotrópica de borda. Esta é derivada da transformação matemática da função $K[L(r)=\operatorname{sqrt}(K(r) / p l)]$, onde $r$ representa os raios crescentes em torno de cada processo pontual e $K$ representa os valores da função $K$, para um determinado valor de $r$, com o objetivo de facilitar a visualização gráfica. Ressalta-se, também, que a função $K$ de Ripley calcula a frequência dos eventos ocorridos em círculos com raio $h$ (ANJOS et al. 2004 apud SANTOS et al. 2016). Testou-se envelopes de completa aleatoriedade espacial, com 999 simulações, a fim de verificar a significância dos padrões observados (agregação ou repulsão) dos sobreviventes da espécie. Para a análise da tendência espacial de primeira ordem, foi utilizada a razão da verossimilhança e o teste de quiquadrado $(p<0,05)$. Na situação de existência de tendência espacial, a qual indica processos nãoestacionários, a função $L$ foi determinada para processos pontuais espaciais não homogêneos. Para os indivíduos sobreviventes, também foi determinada a estimativa de densidade por Kernel. Todas as análises foram realizadas por meio da linguagem de programação estatística $R$ ( $R$ DEVELOPMENT CORE TEAM 2017), junto com os pacotes vegan (OKSANEN et al. 2009) e spatstat (BADDELEY \& TURNER 2005).

\section{RESULTADOS E DISCUSSÃO}

No fragmento florestal, foram amostrados 375 indivíduos, dos quais 99 são pertencentes a espécie Drimys angustifolia (26,4\%). A elevada abundância sugere que a espécie apresenta grande potencial de estabelecimento em áreas altomontanas com influência nebular. Neste sentido, a área do estudo, mesmo apresentando condições limitantes (temperatura, luminosidade, umidade etc.) (KEDDY 1992), é considerada como de elevado potencial para o estabelecimento da espécie. Outros estudos realizados em áreas AltoMontanas também destacam elevada representatividade de $D$. angustifolia (MARTINS et al. 2012, HIGUCHI et al. 2013) associado a espécies da família Myrtaceae e caracterizam esta espécie como indicadora de áreas de altitude (MARTINS-RAMOS et al. 2011, HERTZOG et al. 2016).

A distribuição diamétrica dos indivíduos de $D$. angustifolia não apresentou normalidade de acordo com o teste Shapiro-Wilk $(p<0,05)$, embora pode-se observar, pela Figura 1 , tendência à normalidade. Houve elevado número de indivíduos nas classes intermediárias de diâmetro, principalmente entre 34 a 88 $\mathrm{cm}$, e poucos indivíduos nas maiores classes de diâmetro. SCHAAF et al. (2006), encontraram resultados semelhantes para populações de Ocotea porosa (Nees), com indivíduos distribuídos ao longo de todas as classes diamétricas, e tendência de aumento do número de indivíduos nas classes diamétricas superiores com o amadurecimento da floresta. 


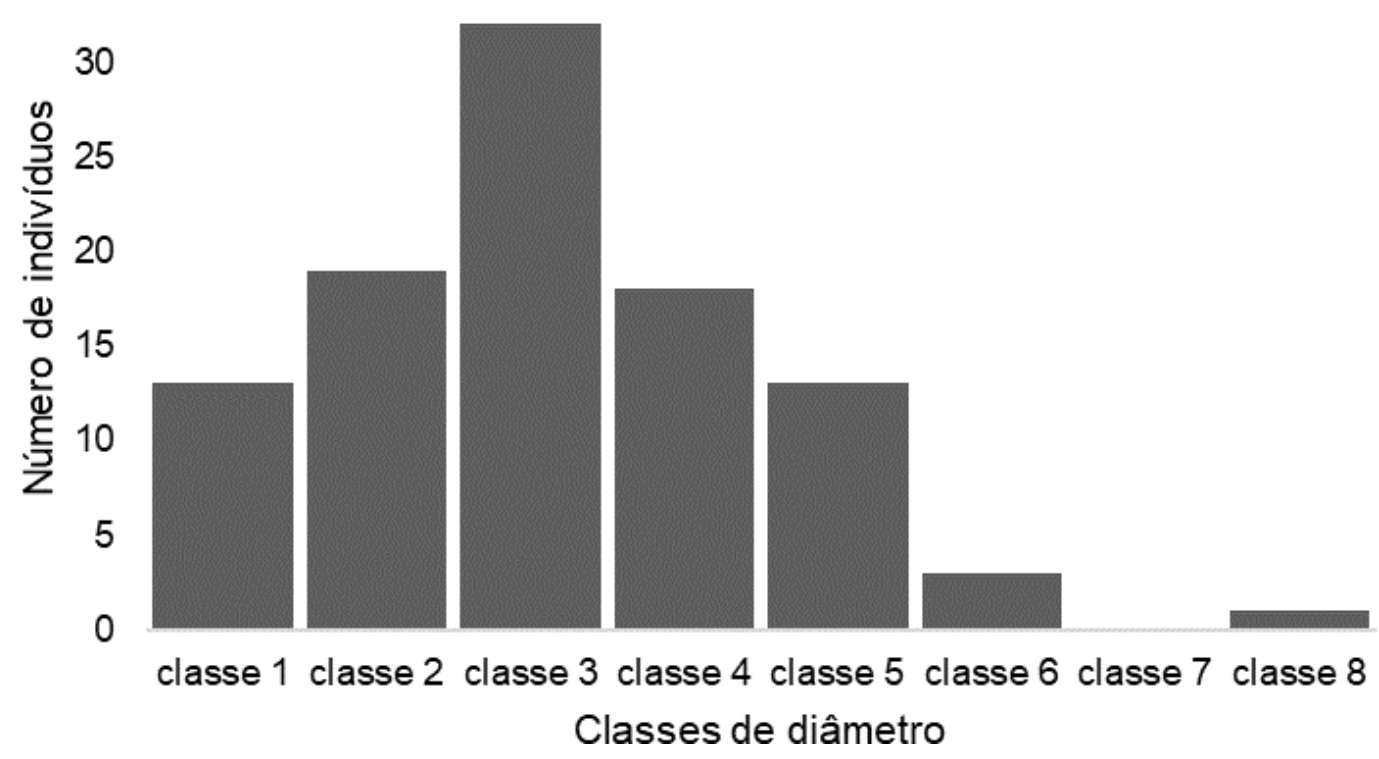

Figura 1. Estrutura diamétrica de Drimys angustifolia em um fragmento de Floresta Ombrófila Mista Alto Montana, Urubici, SC. Classe $1=17$ a $34,87 \mathrm{~cm}$, Classe $2=34,88$ a $52,75 \mathrm{~cm}$, Classe $3=52,76$ a $70,63 \mathrm{~cm}$, Classe $4=70,64$ a $88,51 \mathrm{~cm}$, Classe $5=88,52$ a $106,39 \mathrm{~cm}$, Classe $6=106,40 \mathrm{a}$ $124,27 \mathrm{~cm}$, Classe $7=124,28$ a $142,15 \mathrm{~cm}$, Classe $8=142,16$ a $160,00 \mathrm{~cm}$.

Figure 1. Diametric structure for Drimys angustifolia in a fragment of Upper Montane Mixed Ombrophilous Forest, Urubici, SC. Class $1=17$ to $34.87 \mathrm{~cm}$, Class $2=34.88$ to $52.75 \mathrm{~cm}$, Class $3=52.76$ to 70.63 $\mathrm{cm}$, Class $4=70.64$ to $88.51 \mathrm{~cm}$, Class $5=88.52$ to $106.39 \mathrm{~cm}$, Class $6=106.40$ to $124.27 \mathrm{~cm}$, Class $7=124.28$ to $142.15 \mathrm{~cm}$, Class $8=142.16$ to $160.00 \mathrm{~cm}$.

$\mathrm{Na}$ área em processo inicial de regeneração, foram amostrados 35 indivíduos regenerantes de $D$. angustifolia em 2014 e 42 indivíduos em 2016. Essa mudança em abundância no período de dois anos foi devido ao maior recrutamento $\left(14,28 \%\right.$.ano $\left.{ }^{-1}\right)$ do que mortalidade $\left(2,85 \%\right.$.ano $\left.{ }^{-1}\right)$, indicando que a espécie encontra-se em uma fase de estruturação populacional, apresentando pequeno aumento no número de indivíduos, inerente à característica ecológica da espécie e a fase sucessional em que se encontra a área.

Os 42 indivíduos amostrados em 2016 representam somente 4,07\% do total de indivíduos da comunidade regenerante (1.030 indivíduos). Apesar disso, a tendência de aumento populacional indicado na dinâmica sugere que, ao longo do processo sucessional, pode haver um aumento no número de indivíduos ao longo do tempo. Como a espécie estudada trata-se de uma clímax exigente em luz, que tende a colonizar fases mais tardias durante a sucessão, sugere-se que seu aumento populacional se dará posteriormente, quando a área em estágio inicial estiver em processo sucessional mais avançado. De fato, nota-se que a composição florística-estrutural da comunidade regenerante, atualmente, é formada, predominantemente, por espécies pioneiras típicas, especialmente aquelas pertencentes às famílias Asteraceae e Solanaceae (DALLABRIDA et al. 2017).

A distribuição hipsométrica dos regenerantes de $D$. angustifolia em 2014 não apresentou normalidade $(p<0,05)$ (Figura 2), sendo caracterizada por uma maior concentração de indivíduos nas primeiras classes de altura $(0,65$ a 1,876 m). Em contrapartida, quando se observa a distribuição em altura dois anos depois, é possível detectar distribuição normal $(p>0,05)$ (Figura 3). Dessa forma, houve um acréscimo no número de indivíduo nas classes intermediárias de altura e redução nas menores classes, em relação ao ano de 2014. Provavelmente esse padrão ocorreu devido ao crescimento em altura dos indivíduos menores de 2014, que passaram a ter uma estatura maior em 2016, acompanhado pelo baixo recrutamento de indivíduos de menores tamanhos.

A população de $D$. angustifolia do fragmento florestal apresentou distribuição espacial aleatória, com valor do Índice de Morisita de 1,121 (limite superior 1,141, inferior 0,897). A distribuição aleatória indica que não há tendência dos indivíduos se agruparem e nem ocorrerem de forma uniforme. Esse padrão pode sugerir diferentes características ecológicas ou de ocupação da espécie e, segundo RICKLEFS \& MILLER (2000), está relacionado a ausência de pressões antagônicas (e.g. competição intra-específica) ou restrições de distribuições (e.g. preferência por hábitat). 


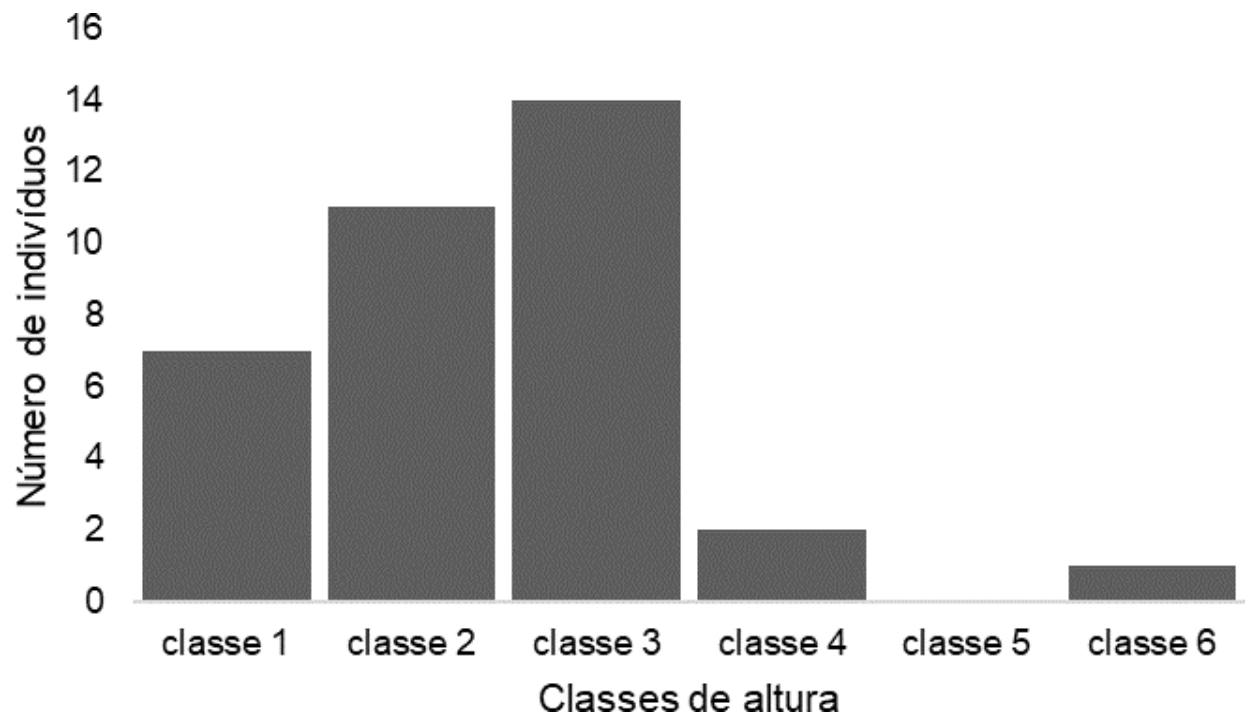

Figura 2. Estrutura hipsométrica de Drimys angustifolia em 2014, em uma área em processo de restauração em Urubici, SC. Classe $1=0,65$ a $1,058 \mathrm{~m}$; Classe $2=1,059$ a 1,467 m; Classe $3=1,468$ a 1,876 $\mathrm{m}$; Classe $4=1,877$ a 2,285 m; Classe $5=2,286$ a 2,694 m; Classe $6=2,695$ a 3,10 m.

Figure 2. Hypsometric structure of Drimys angustifolia in 2014, in an area undergoing restoration in Urubici, SC. Class $1=0.65$ to $1.058 \mathrm{~m}$; Class $2=1.059$ a $1.467 \mathrm{~m}$; Class $3=1.468$ to $1.876 \mathrm{~m}$; Class $4=$ 1.877 to $2.285 \mathrm{~m}$; Class $5=2.286$ to $2.694 \mathrm{~m}$; Class $6=2.695$ to $3.10 \mathrm{~m}$.

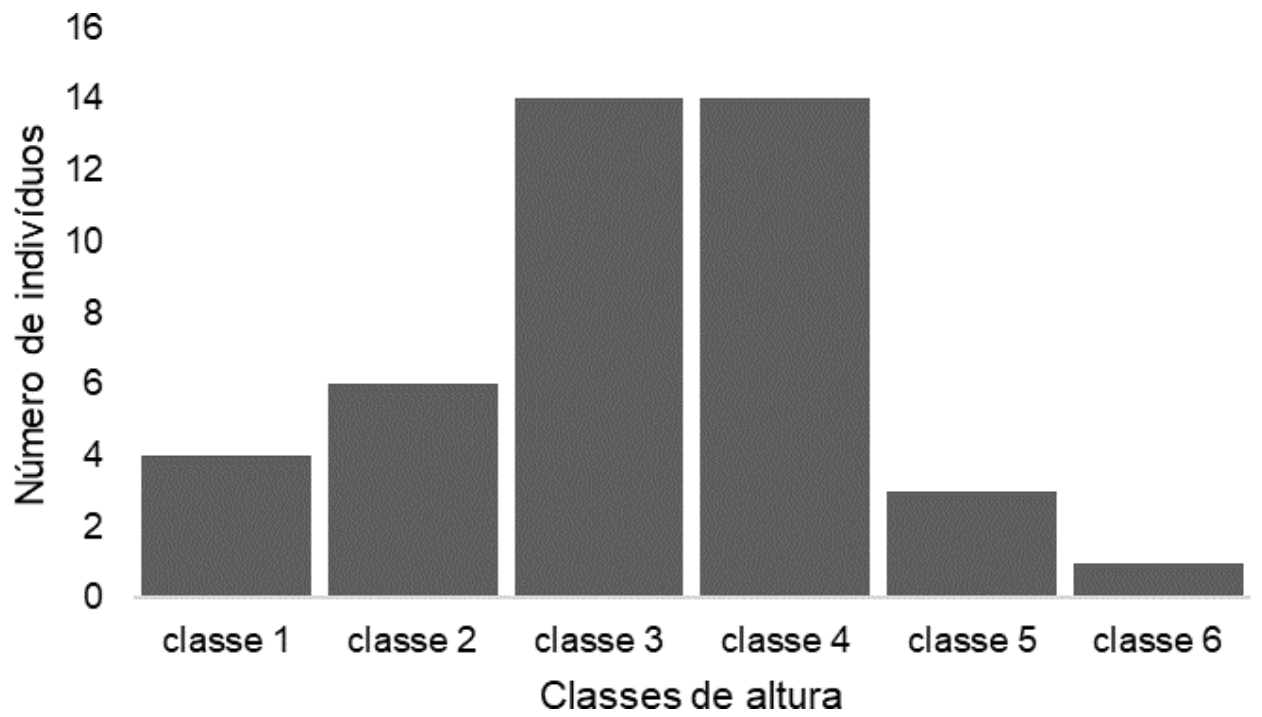

Figura 3. Estrutura hipsométrica de Drimys angustifolia em 2016, em uma área em processo de restauração em Urubici, SC. Classe $1=0,5$ a 0,941 m; Classe $2=0,942$ a 1,383 m; Classe $3=1,384$ a 1,825 m; Classe $4=1,826$ a 2,267 m; Classe $5=2,268$ a 2,709 m; Classe $6=2,710$ a $3,15 \mathrm{~m}$.

Figure 3. Hypsometric structure of Drimys Angustifolia in 2016, in an area undergoing restoration in Urubici, SC. Class $1=0.500$ to $0.941 \mathrm{~m}$; Class $2=0.942$ to $1.383 \mathrm{~m}$; Class $3=1.384$ to $1.825 \mathrm{~m}$; Class $4=$ 1.826 to $2.267 \mathrm{~m}$; Class $5=2.268$ to $2.709 \mathrm{~m}$; Class $6=2.710$ to $3.15 \mathrm{~m}$.

$\mathrm{Na}$ área em recuperação, os indivíduos regenerantes da espécie, no ano de 2016, apresentaram um padrão de distribuição espacial agregado, com Índice de Morisita de 1,324 (limite superior 1,266 e inferior $0,824)$, sendo que a maior densidade de indivíduos foi nas parcelas mais próximas do remanescente florestal. Esse padrão de distribuição pode estar relacionado ao histórico de degradação da área, condições ambientais locais, padrões de dispersão, e às características ecológicas da espécie. NASCIMENTO et al. (2001), em estudo dos padrões de distribuição espacial de espécies em um fragmento de Floresta Ombrófila Mista, verificaram que $46,7 \%$ das espécies apresentaram padrão de distribuição agregado, e destacaram este padrão como sendo resultado da elevada quantidade de indivíduos de pequeno porte compondo a 
estrutura do floresta, após distúrbios. Além disso, seu agrupamento possivelmente ocorre por fatores que dependem da qualidade do sítio, dispersão zoocórica e reprodução (BORCARD et al. 1992, PERRY \& DIXON 2002). Provavelmente, a distribuição agregada próxima ao remanescente florestal está relacionada, também, à maior proximidade com a fonte de propágulos.

A distribuição espacial dos indivíduos mortos, recrutas e sobreviventes do componente regenerativo, no período de 2014 a 2016, está indicado na Figura 4a. O gradiente da escala em cinza indica que as áreas mais escuras possuem maior probabilidade de ocorrência de indivíduos sobreviventes de $D$. angustifolia. Observa-se que a maior densidade de indivíduos está localizada nas parcelas próximas ao fragmento florestal, sugerindo que condições ambientais (e.g. condições mais favoráveis próximas ao fragmento) ou fatores de dispersão (e.g. dispersão próximo à fonte de propágulos, considerando como a fonte os indivíduos adultos do fragmento florestal) sejam os fatores determinante na estruturação das populações da espécie no local. Embora a distribuição espacial dos indivíduos sobreviventes indique uma tendência de agregação nas parcelas próximas à borda do fragmento, a análise da distribuição espacial de segunda ordem, por meio da função $L$, evidenciou que os indivíduos se distribuíram de forma aleatória entre si na área avaliada (Figura 4b), indicando a ausência de atração ou repulsão entre os mesmos.
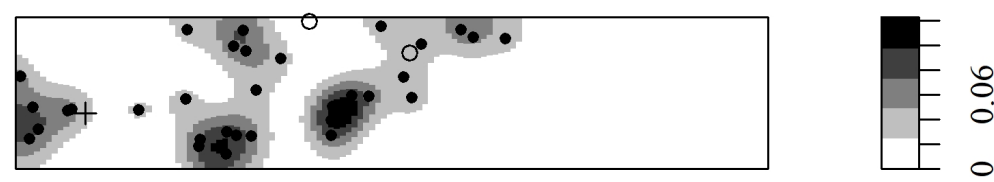

a

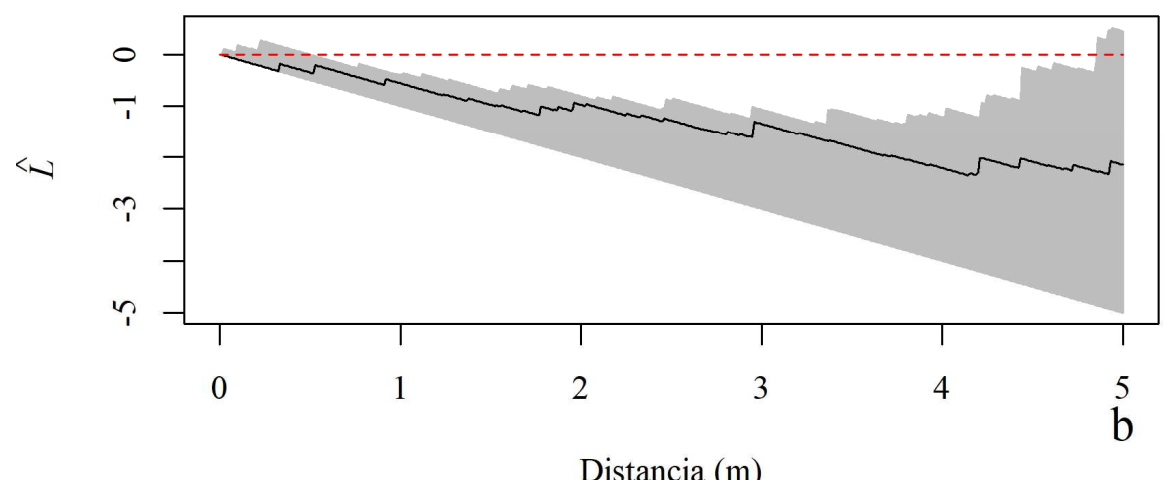

Figura 4. Distribuição espacial dos indivíduos sobreviventes (círculos pretos), mortos (cruz) e recrutas (círculos abertos) de Drimys angustifolia, com estimativa da densidade dos sobreviventes por Kernel (a). O fragmento florestal encontra-se à esquerda da transecção aqui representada. Função $L$ (b) para processos espaciais não homogêneos, com correção isotrópica, para os indivíduos sobreviventes de $D$. angustifolia em área de campo em estágio inicial de regeneração no Parque Nacional de São Joaquim, em Urubici, Santa Catarina. A linha preta representa o valor de $L$ observado. O envelope cinza representa uma relação espacial de completa aleatoriedade. Valores de $L$ acima do envelope cinza indica associação espacial positiva (atração). Valores de $L$ abaixo do envelope cinza representa associação espacial negativa (repulsão).

Figure 4. Spatial distribution of surviving individuals (black circles), death (cross) and recruits (open circles) of Drimys angustifolia, with estimated density of survivors per kernel (a). The forest fragment is to the left of the transection represented here. Function $L(b)$ for non-homogenous space processes, with isotropic correction, for the survivors individuals of $D$. angustifolia in a field area in an initial stage of regeneration in the national park of São Joaquim, in Urubici, Santa Catarina. The black line represents the observed $L$ value. The gray area represents a spatial relation of complete randomness. $L$ values above the gray letter indicate positive (attraction) space association. $L$ values below the gray area represents negative spatial association (repulsion). 


\section{CONCLUSÃO}

A espécie mais abundante no fragmento estudado foi Drimys angustifolia, que apresentou diferentes padrões de estrutura populacional entre os componentes adulto e regenerante. Enquanto o componente adulto no fragmento florestal se destacou pela elevada abundância, a espécie foi pouco representativa no componente regenerativo na área adjacente, em recuperação pós-distúrbio. No entanto, foi constatado incremento do número de indivíduos regenerantes ao longo dos dois anos de observação, indicando tendência ao aumento populacional ao longo do processo sucessional. Os padrões de distribuição em classes de diâmetro e altura também se diferenciaram entre os estratos adulto e regenerante, com distribuição normal somente para o histograma da distribuição hipsométrica do componente regenerante de 2016. Além disso, também foram encontrados padrões de distribuição espacial distintos entre os componentes. Esses padrões são esperados considerando que, em geral, os estratos podem se diferenciar estruturalmente com a idade da planta. A elevada densidade desta espécie, associada às suas características estruturais, indicam boa adaptação no fragmento florestal e capacidade regenerativa condizente com o estágio sucessional da área em recuperação, com tendência ao aumento do número de indivíduos ao longo do tempo.

\section{REFERÊNCIAS}

BADDELEY A \& TURNER R. 2005. Spatstat: an R package for analyzing spatial point patterns. Journal of Statistical Software 12: 1-42.

BECKER A et al. 2006. Ecological and land use studies along elevational gradients. Mountain Research and Development 27: 58-65.

BOLSTAD PV \& VOSE JM. 2005. Forest and pasture carbon pools and soil respiration in the southern Appalachian Mountains. Forest Science 51: 372-383.

BORCARD D et al. 1992. Partialling out the spatial component of ecological variation. Ecology 73: 1045-1055.

BRUIJNZEEL LA. 2000. Hydrology of tropical montane cloud forests: a reassessment. In: GLADWELL JS. (Ed.). Proceedings of the Second International Colloquium: 353-383.

DALE MRT. 2000. Spatial pattern analysis in plant ecology. Cambridge: University press. 326p.

DALLABRIDA JP et al. 2017. Elementos nucleadores da paisagem influenciam a dispersão zoocórica em áreas campestres alto-montanas. Rodriguésia 68: 325-335.

FALKENBERG DB. 2003. Matinhas nebulares e vegetação rupícola dos Aparados da Serra Geral (SC/RS), sul do Brasil. Tese (Doutorado em Biologia Vegetal). Campinas: UNICAMP. 594p.

HERTZOG A et al. 2016. Winteraceae do Rio Grande do Sul, Brasil. Rodriguésia 67: 251-260.

HIGUCHI P et al. 2013. Florística e estrutura do componente arbóreo e análise ambiental de um fragmento de Floresta Ombrófila Mista Alto-Montana no município de Painel, SC. Ciência Florestal 23: 153-164.

IBGE. 2012. Manual técnico da vegetação brasileira. 2.ed. (Série: Manuais técnicos em geociências, n.1). Rio de Janeiro: IBGE. 275p.

KEDDY PA. 1992. Assembly and response rules-2 goals for predictive community ecology. Journal of Vegetation Science 3: 157-164.

KLEIN RM. 1978. Mapa fitogeográfico do Estado de Santa Catarina. Flora llustrada Catarinense: 24p.

MARTíNEZ ML et al. 2009. Effects of land use change on biodiversity and ecosystem services in tropical montane cloud forests of Mexico. Forest Ecology and Management 258: 1856-1863.

MARTINS-RAMOS D et al. 2011. Florística de Floresta Ombrófila Mista Altomontana e de Campos em Urupema, Santa Catarina, Brasil. Revista Brasileira de Biociências 9: 156-166.

MARTINS D et al. 2012. Estrutura de um remanescente de Floresta Ombrófila Mista em Urupema. Revista de Ciências Agroveterinárias 11: 126-137.

MYERS N et al. 2000. Biodiversity hotspots for conservation priorities. Nature 403: 853-845.

NASCIMENTO ART et al. 2001. Estrutura e padrões de distribuição espacial de espécies arbóreas em uma amostra de Floresta Ombrófila Mista em Nova Prata, RS. Ciência Florestal 11: 105-119.

OKSANEN J et al. 2009. Vegan: community ecology package. R package version 1: 8.

PERRY JN \& DIXON PM. 2002. A new method to measure spatial association for ecological count data. Ecoscience 9: 133-141.

R DEVELOPMENT CORE TEAM. 2017. R: A language and environment for statistical computing. R Foundation for Statistical Computing. Disponível em: http://www.R-project.org. Acesso em: 02 jun. 2017.

RICKLEFS RE \& MILLER GL. 2000. Ecology. 4.ed. New York: W.H. Freeman and Company.

SANTOS AP et al. 2016. Avaliação da acurácia posicional em dados espaciais utilizando técnicas de estatística espacial: proposta de método e exemplo utilizando a norma brasileira. Boletim de Ciências Geodésicas 22: 630-650.

SCHAAF LB et al. 2006. Alteração na estrutura diamétrica de uma Floresta Ombrófila Mista no período entre 1979 e 2006. Revista Árvore 30: 283-295.

SCHEER MB et al. 2011. Funcionalidades ambientais de solos altomontanos na Serra da Igreja, Paraná. Revista Brasileira de Ciência do Solo 35: 1113-1126. 
SHEIL D \& MAY RM. 1996. Mortality and recruitment rate evaluations in heterogeneous tropical forests. Journal of Ecology 84: 91-100.

SILVA AC et al. 2012. Relações florísticas e fitossociologia de uma Floresta Ombrófila Mista Montana Secundária em Lages, Santa Catarina. Ciência Florestal 22: 193-206.

SIMINSKI A et al. 2004. Sucessão florestal secundária no município de São Pedro de Alcântara, litoral de Santa Catarina: estrutura e diversidade. Ciência Florestal 14:21-33.

SIMINSKI A et al. 2013. Classificação da vegetação secundária em estágios de regeneração da Mata Atlântica em Santa Catarina. Ciência Florestal 23: 369- 378.

VIBRANS AC et al. 2011. Structure of mixed ombrophyllous forests with Araucaria angustifolia (Araucariaceae) under external stress in Southern Brazil. Revista de Biologia Tropical 59: 1371-1387. 